\title{
Extracorporeal Membrane Oxygenation and Temperature Management in Postresuscitation
}

\author{
Moderator: Hans Friberg, $\mathrm{MD}^{1}$ \\ Participants: Jonathan Paul, MD, ${ }^{2}$ Graham Nichol, MD, MPH, FAHA, and Takahiro Nakashima, MD ${ }^{4}$
}

\begin{abstract}
During the 2018 Therapeutic Hypothermia and Temperature Management meeting in Miami, three state-of-the-art lectures were presented on the current advances in the use of therapeutic hypothermia and targeted management in selected patient population. Dr. Hans Friberg from Lund University moderated this very informative discussion. Dr. Jonathan Paul, University of Chicago, presented information on methods on advance resuscitation using extracorporeal membrane oxygenation (ECMO) as a beneficial technique in providing prolonged cardiac and respiratory support to persons with cardiac arrest. Dr. Paul emphasized the potential consequences of bleeding even when cooling at $33^{\circ} \mathrm{C} . \mathrm{Dr}$. Graham Nichol, University of Washington-Harborview Center in Seattle, discussed strategies to rewarming after accidental hypothermia that seem to be helpful for the patient's recovery. Finally, Dr. Takahiro Nakashima from the National Cerebral and Cardiovascular Center, Suita, Japan, spoke on the ECMO and hypothermia Japanese trial results, he emphasized the time window of effective resuscitation and the importance of bystander cardiopulmonary resuscitation $(C P R)$ and early implementation of extracorporeal CPR $(E C P R)$ with patients who had refractory out of hospital cardiac arrest via ECPR and advantages over conventional CPR. Together these presentations provided a rich background for a very informative question and answer session at the meeting.
\end{abstract}

Question: Within my institution, with cardiac arrest, they have needed ECMO, and then, they are inducing targeted temperature management (TTM). Early on, we did $33^{\circ} \mathrm{C}$, but as the bleeding complications got highlighted with those ECMO specialists, they chose to do $36^{\circ} \mathrm{C}$. Have any of you had bleeding complications when you take these patients on ECMO to $33^{\circ} \mathrm{C}$, which are significant complications?

Dr. Jonathan Paul: At least from our perspective with percutaneous ECMO, bleeding is huge. That is the main complication that we see with or without hypothermia. So, certainly, we have seen significant bleeding with $33^{\circ} \mathrm{C}$. It is hard to say, because the numbers are small whether or not that bleeding would have occurred at $36^{\circ} \mathrm{C}$ or at normothermia. I don't have enough numbers to say whether $33^{\circ} \mathrm{C}$ versus $36^{\circ} \mathrm{C}$ will have a better bleeding profile but bleeding is the number one cause of morbidity with ECMO.

Dr. Takahiro Nakashima: In our institution, we routinely manage at $34^{\circ} \mathrm{C}$, but as you said, patients with ECMO have bleeding complications (in about $50 \%$ of the patients) according to Japanese studies. The leading site of bleeding is the puncture site, in about $25 \%$. So, we ask the surgeon to suture, and if the bleeding has not stopped, we often stop $34^{\circ} \mathrm{C}$ hypothermia and change to $36^{\circ} \mathrm{C}$ normothermia management.

Dr. Graham Nichol: I think the merits of $33^{\circ} \mathrm{C}$ versus $36^{\circ} \mathrm{C}$ to reduce brain injury postcardiac arrest are warranted. Our approach to management of anticoagulation in patients on mechanical circulatory support is to use heparin dosing guided by anti-10A level rather than guided by partial thromboplastin time (PTT). Our anecdotal experience is that we have fewer bleeding complications.

Dr. Hans Friberg: What is the typical temperature at the time of initiation of extracorporeal life support in patients with refractory cardiac arrest?

Dr. Jonathan Paul: Just anecdotally, it is usually at $35^{\circ} \mathrm{C} /$ $36^{\circ} \mathrm{C}$ range from what I have seen.

Dr. Graham Nichol: Postarrest, most patients come in around $35^{\circ} \mathrm{C}$.

Dr. Hans Friberg: Yes, we had $35.3^{\circ} \mathrm{C}$ as the median admission temperature in the TTM 1 trial. But I thought that here it is longer times to the targeted temperature, 55 minutes up to 1 hour before cannulation. Do you monitor temperature online with the devices?

Dr. Jonathan Paul: There is actually a warmer built into the system that we use. In fact, we are probably doing counter to what we are proposing here by rewarming the patients (with the ECMO circuit) to bring them back to normal temperature.

\footnotetext{
${ }^{1}$ Department of Anesthesiology and Intensive Care Medicine, Skane University Hospital, Lund University, Lund, Sweden.

${ }^{2}$ Section of Cardiology/Interventional Cardiology, University of Chicago Medicine, Chicago, Illinois.

${ }^{3}$ University of Washington-Harborview Center for Prehospital Emergency Care, Division of Internal Medicine, Seattle, Washington.

${ }^{4}$ Department of Cardiovascular Medicine, National Cerebral and Cardiovascular Center, Suita, Japan.
} 
I think that there is some give and take with the perfusionist and trying to come up with a middle ground.

Question: Dr. Nichol, I saw that you highlighted what you use for heparin dosing in this indication, which included a nomogram for weight-based heparin dosing. I was interested since we are all talking about bleeding complications if anyone on the panel other than using anti-10A has experimented with using less heparin if you are going to take them to $33^{\circ} \mathrm{C}$, perhaps that nomagram is too much heparin.

Dr. Graham Nichol: It may be that lower doses of heparin are safe and effective. One of the challenges is that it is very hard to do randomized trials on patients who are relatively rare and present sporadically over time. Every center has its own recipe for anticoagulation. A lot of it is based on Gestalt or experience. Dr. Marko Noc from Slovenia has a lot of experience with ECMO after cardiac arrest, maybe he could comment on his approach to anticoagulation.

Dr. Marko Noc: I think that this has been a great session, and ECPR patients are nowadays indeed the ones we need to discuss. I agree with you, that in addition to infection, ECMOrelated bleeding is a serious problem. But since we developed a protocol with fluoroscopy-guided percutaneous implantation in the catheterization laboratory, we have been able to minimize the bleeding at the cannula site. In the cath lab we also implant a distal limb perfusion sheath and connect to the arterial cannula port. We learned it is better to do it upfront before placing the arterial cannula to avoid limb ischemia, which typically develops once you get to the cardiac care unit (CCU). In terms of bleeding in ECPR patients, one has to keep in mind that these are patients with 1 hour or even more of ongoing mechanical chest compressions with a device such as "Lucas" or "AutoPulse." Because of low flow during chest compression, one typically does not see possible resuscitation-related bleeding before restarting organ perfusion with the ECMO. It is therefore our strategy to go from the cath lab directly to the CT scan of the head, thorax, and abdomen to document hopefully no brain edema and get the answer about possible resuscitation-related bleeding and injuries such as hemothorax, liver, or spleen rupture. Early diagnosis makes it possible to solve it before profound hemorrhagic shock develops.

Dr. Graham Nichol: Can I ask you to comment further? Is it your sense that your lower rate of bleeding problems is because that your group now has experience with technique or the drugs you use?

Dr. Marko Noc: I think less bleeding is primarily related to percutaneous implantation which is done by individuals who regularly do this in the cath lab. Since vessel punctures are part of every-day life for interventional cardiologist, it is much easier for them to operate also under ongoing chest compression without palpable pulse. Another reason for less bleeding may be related to more thoughtful use of unfractionated heparin (UFH), which we dose according to PTT (target $\leq 50$ seconds). In case of significant bleeding, we would of course stop it. With newer ECMO cannulas and membranes, we are not so worried anymore about short ECMO runs without optimal anticoagulation.
Dr. Graham Nichol: So, you are running your patients initially without heparin?

Dr. Marko Noc: After we place the cannulas, we administer a bolus of $5000 \mathrm{U}$ of UFH. Before connecting cannulas to the ECMO console, we allow back-bleeding to remove any possible clot. After the start of ECMO, we go on and perform coronary angiography. If this is followed by percutaneous coronary intervention (PCI), a patient receives an additional bolus of UFH according to activated clotting time (ACT), which we would like to see above 250 seconds. If the patient gets a coronary stent, we administer $250-500 \mathrm{mg}$ acetylsalicylic acid intravenously, and P2Y12 inhibitor (i.e., ticagrelor) as crushed tablets via nasogastric tube. Based on our own randomized trial, we use ticagrelor rather than clopidogrel because there is essentially no platelet inhibition after clopidogrel loading for the first 48 hours. Because of the threat of profound bleeding, we absolutely avoid periprocedural GP 1lb/1lla inhibitors such as abciximab and eptifibatide. After transfer of the patient to the CCU, and if their CT scan has not shown any significant bleeding, we measure PTT and start UFH infusion when $<50$ seconds are reached.

Dr. Hans Friberg: Marko, what is the temperature that you use in your circuit?

Dr. Marko Noc: We are still somewhere between $32^{\circ} \mathrm{C}$ to $34^{\circ} \mathrm{C}$. We cool those patients.

Dr. Michael Kurz: That is a very important point. Could I just make sure that that is clear. So, you take the patient to the cath lab, you give him a slug of heparin to get to whatever ACT you want, you then cannulate them, fix whatever stent you need, and then from there you go what I understood as days without giving anymore heparin?

Dr. Marko Noc: We do as I described previously. There is not much data on anticoagulation/antiplatelet treatment in ECPR patients undergoing concomitant PCI who have, as we discussed also strikingly increased risk of bleeding. And there is probably also increased a risk of stent thrombosis, another life-threatening complication, which we did not discuss. Admittingly, however, our anticoagulation/antiplatelet strategy may be, because of the lack of adequate studies, regarded as an "educated guess.",

Comment: What antiplatelet drugs are you using?

Dr. Marko Noc: As I pointed out, if we place a stent, we would use an intravenous bolus of acelysalicilic acid (250$500 \mathrm{mg}$ ) followed by $100 \mathrm{mg}$ iv per day, and loading dose of ticagrelor $(180 \mathrm{mg})$ as crushed and dissolved tablets administered via nasogastric tube followed by $90 \mathrm{mg}$ bid.

Dr. Jonathan Paul: I think that this is useful information. One question I have is, what are the typical sizes of cannulas that you are using, because as the cannula size goes down, we know the flow goes down. Lower flows will probably lead to higher rates of thrombosis as well. We typically use a 15-17 arterial and a 21-25 venous, but I would be curious to hear what your standard is. 
Dr. Marko Noc: If you really want to have good flow then you increase the venous cannula. For example, we would go for $25 \mathrm{~F}$, but some centers go also to $29 \mathrm{~F}$. For arterial side, we would use 17-19F. Again I would like to emphasize the importance of upfront placement of distal perfusion sheath to avoid ipsilateral limb ischemia.

Question: We have used ACT only postinsertion and I was wondering what the experience of not using PTT, I am intrigued by the anti-10A, but that is what we have been using point of care, ACT throughout the entire time that ECMO is in place. I wonder what the panel thinks about that or any suggestions they may have?

Dr. Jonathan Paul: We typically use both. We use ACT and PTT primarily, but the perfusionist will manage the anticoagulation with PTT goals of around $60-80$. We do not generally use ACT, unless there are issues in interpreting the other anticoagulation markers.

Question: Of course bleeding is the most frequent complication, but we have to be a little careful especially if the cannula stays in place so long. I have had a couple of patients with flu on ECMO who developed ischemic strokes, and if that happens, you lose any chance of good outcome. I would be concerned to not give any kind of coagulation if the catheter is staying in for a long period of time. Dr. Paul, I missed in your protocol, the use of phosphodiesterase inhibitors, I was wondering if there was a reason for that.

Dr. Jonathan Paul: No, we do use phosphodiesterase inhibitors frequently. We find that anecdotally that there are less tachyarrhythmias with these drugs.

Dr. Hans Friberg: Dr. Nakashima, in your study how many of these patients were initiated ECPR, how many had return of spontaneous circulation (ROSC)? Or how many did not? How long would you continue ECMO without ROSC?

Dr. Takahiro Nakashima: Our study did not include those patients who achieved ROSC before hospital arrival or initiation of ECPR. The rate of ROSC is $70 \%$ in ECPR group and $30 \%$ in conventional CPR (CCPR) group.

Dr. Hans Friberg: In those who did not have ROSC, when do you withdraw ECPR in those patients?

Dr. Takahiro Nakashima: It depends on the patient.

Dr. Hans Friberg: What is the official view on that as these are potential organ donors, and even those with ROSC with extensive brain damage are also potential organ donors. We have not really discussed that issue today, but it definitely is an important issue with this technique.

Dr. Takahiro Nakashima: In our institution, we keep patients on ECPR for a median of 7 days. Within 7 days, if the patient has not regained ROSC, we stop the treatment.

Dr. Marko Noc: In our experience, a great majority of patients get ROSC within a few hours after ECMO start, in particular, if the cause is acute coronary occlusion resolved by immediate PCI. If ROSC is not achieved for several hours, this might be a real problem. Since there is no anterograde flow through the left ventricle/across aortic valve, left ventricle will gradually distend, pulmonary edema will develop, and ultimately the cavity may thrombose. Therefore, for example, if the patient had no ROSC on one hand and very rapid neurological recovery on the other hand, he would be a candidate for LVAD/BiVAD or urgent heart transplant before lethal LV thrombosis develops.

Dr. Hans Friberg: So, in your experience, how often do patients not have ROSC.

Dr. Marko Noc: I think it is up to $20-30 \%$, but of course, it depends upon your patient selection.

Question: I know of at least one case where the reversal of myocardial stunning took a couple of days. Is there a guideline or anything in terms of how long to go before the declaration of death?

Dr. Hans Friberg: Does it say in any guidelines how long? We have not touched on that.

Dr. Graham Nichol: I think it is based on local protocol.

Question: What is the panel's experience of the longest you have gone in seeing a reversal of the myocardial stunning?

Dr. Jonathan Paul: I think your experience is consistent with what we see. If we don't see recovery after the postresuscitation dysfunction within 2 days, it is not going to get any better at least in my experience. It has been interesting that I have heard about other protocols where patients are put on ECMO within 30 minutes of coming into the emergency room (ER) and before returning the patient to the intensive care unit (ICU), they will send the patient to the CT scan mainly to look for injury to the brain. If there are signs of irreversible brain damage on initial imaging, you would decannulate or just turn off ECMO before moving the patient to the ICU. Keeping a patient on ECMO for 7 days I think is too long. It is hard on the family when there is probably no hope. I think avoiding that is really important.

Dr. Marko Noc: I would also emphasize the role of bedside echocardiography. When the heart is recovering, you have to monitor that very carefully. Try to reduce the retrograde ECMO flow to decrease LV afterload, thereby creating optimal hemodynamic situation to assess possible recovery of LV contractility and thereby speed up your ECMO weaning. And when doing such maneuvers, you need echo to see what the heart is doing. The worst thing is to get the patient to the $\mathrm{CCU}$, set up retrograde ECMO flow to 4-5 L/min, and enjoy return of urine output and lactate drop without seeing what the heart is doing.

Dr. Hans Friberg: So controlled studies are one thing, but then you have the clinical reality too. I would be interested in hearing the panels view on that.

Dr. Graham Nichol: We do ECMO for refractory cardiac arrest on a selective basis. We have an agreed-upon criteria but each patient requires a discussion between the emergency 
physician and surgeon. It is handled on a case by case basis. It is not perfect. I think that is how it happens in most centers.

Dr. Jonathan Paul: We struggle with this as well. This is a daily conversation we have in the cath lab. Depending on the provider in the ER, the question is often something like "you had a patient yesterday you took for ECMO, why won't you take this patient for ECMO?' It really is a case by case discussion. We really use the criteria set forth in the American College of Cardiology (ACC) article. If a patient has a downtime of 60 minutes and the lactate is 12 and the $\mathrm{pH}$ is 6.9, we are not going to take that patient to the cath lab because we know that the outcome is going to be incredibly poor. I do not think there is any hard formula for these cases.

Dr. Takahiro Nakashima: We would do ECPR for refractory cardiac arrest. We usually consider time from collapse to ROSC and cardiac arrest rhythm for ECPR use, only time and rhythm, because most patients transported to our institution have the reversible cause of cardiac arrest.

Dr. Graham Nichol: In our center, it is evolving fairly quickly. In essence, our criteria are as follows: hypothermic arrest, (i.e., environmental exposure), someone who is "young" and has a reversible arrest so prolonged ventricular fibrillation (VF), or a high index of suspicion of massive pulmonary emboli.

Comment: I think in addition to what Dr. Nichol mentioned at our institute, we do have an ECPR program that we are starting as well. A problem at our institution is that we do not have cardiothoracic surgeons on site. If we are going to go on doing an assist device such as an LVAD or something else that needs to be done, that patient has to be transported to another hospital, so now you have a conversation between three people in terms of the management of these patients. We do not progress to that rapidly because we will do temperature management and ECMO for 48 hours and see where that patient is neurologically and make a decision then to see if they go on to the other facility. I think in terms of doing this, it does take a team and you really have to have the criteria set up ahead of time. Part of the problem is that sometimes we do not always have the right information. For example, I had a patient whom I put on VA ECMO, who supposedly suffered a refractory cardiac arrest, and I was told that the patient was 55 years of age, but by the time we actually found out the true age of the patient, didn't know this because the patient was under drapes, he was getting a cath at the time, and the patient turned out to be 85 years of age. So here, we are putting VA ECMO cannula into someone who is 85 , and who we know is not going to recover. Sometimes we do need the best information as possible.

Dr. Jonathan Paul: I think with regard to the rhythm question, pulseless electrical activity (PEA) has always been regarded as an absolute contraindication but that is not necessarily what it should be especially with pulmonary embolism. That is the big patient population that I think we may be missing. It is clearly a reversible cause. The techniques that are available in the cath lab to deal with this are quite advanced. We can deliver catheter directed thrombolytic therapy as well as many other thrombectomy de- vices. Those are the ones that we are really aggressive with, and they tend to do very well with ECMO support.

Dr. Graham Nichol: I have two emergency medicine colleagues who are survivors of massive pulmonary embolus that were treated successfully with ECMO. I think to extend what has been said previously, it is important to recognize that ECMO is clearly transformative for some patients, but it is a means and not an end. You need to have a clear understanding of what your destination is or what your exit is, otherwise the patient will not do well, and it will create a lot of angst and turmoil at your institution.

Question: Dr. Paul, you showed a video where CPR was being done mechanically, could you comment on working around the device while it is in operation because it would appear that perhaps there are some windows not available that you would normally use and how you accomplish that with fluoroscopy with the device in place?

Dr. Jonathan Paul: It is not easy. The more we have done, the better we have gotten. Typically, with the Lucas device, we are able to get cranial views, mostly which are enough to see the left anterior descending artery (LAD), typically in a big ST-segment elevation myocardial infarction. That is about it. With the AutoPulse device, we have had a little more success moving the camera around because it is a smaller device. A challenge with the latter is that a lot of the circuitry that is within the back part of the device interferes with the pictures. There is not a perfect answer. We have had better success using angiography with the Lucas device. We usually work with one or two views and it is usually enough.

Question: So, do you feel like you get an adequate look at the right side? Or do you have to adjust your approach if you have to intervene on the right side?

Dr. Jonathan Paul: We can get a good look at all three major coronary arteries. Typically, if the patient is arresting, it is a left main or a prox LAD, and those are the ones that we can see well. We can see the right coronary artery as well, it just takes a little practice to find the right view.

Question: I am wondering what systems you are putting in place, obviously not every emergency department can do ECMO for CPR, so in some of your large cities, are you advocating for your EMS systems to bypass certain hospitals to go to only certain hospitals?

Dr. Graham Nichol: That is a great question. It really depends on your local circumstance. There is a lot of interest in setting up ECMO programs, but I think they are largely confined to STEMI centers. I think that is, in part, because that is where the expertise is, but it really is going to depend on your local environment.

Dr. Hans Friberg: In our University hospital, the thoracic surgeons do not want to be near this. I know the reason is that they do not want to crowd their thoracic ICUs. There is a flow of patients that would be affected. I know of several sites in Europe where these patients are not primarily in the thoracic ICUs of University Hospitals but in other hospitals without 
thoracic ICUs, where the patients are kept in regular ICUs. This may differ to the United States.

Dr. Graham Nichol: It is the same set of issues in some centers in the United States. I think that some of the cardiothoracic surgeons are concerned that patients who are put on ECMO will impair their ability to do elective cases.

Comment: I think that this decision process of where a patient is taken is important. Dr. Nichol presented in his presentation two individuals who experience the same amount of time in the cold water were taken to two different hospitals. One seemed to be treated appropriately but the other individual was treated differently at the other hospital. How do we get the word out that there are more strategies in this case of controlled rewarming that can be helpful for patients. Where are we with that?

Dr. Graham Nichol: I think it is happening. There are reports in the press of dramatic saves of people with prolonged cardiac arrest who were resuscitated. The case I described was on the front page of our local newspaper. I think there is a lot of pent-up enthusiasm in emergency medicine. The growth of ECMO is astonishing. I think that is, in part, because of these successfully treated cases and, in part, because of the saves of massive pulmonary embolism (PE) and driven by evolution of technology. There are also case series or case reports of ECMO having an impact on patients with severe influenza complicated by acute respiratory distress syndrome (ARDS). Unfortunately, I think we are probably going to have the pendulum swing back a little. I think in some sense that may be appropriate. It is probably not appropriate for every center to have an ECMO program. Other issues to consider include how to obtain rapid vascular access, how to manage ECMO-related complications, and what the destination therapy is.

Dr. Hans Friberg: We will see where the pendulum stops.

Dr. Jonathan Paul: One other quick point about your question. We have done sessions in Chicago Firehouses for first responders to let them know where ECMO is available, and we have found that to be a successful strategy.

Comment: I will just add then that maybe that there is a benefit to case reports. Some journals discourage them but for the Journal of Therapeutic Hypothermia and Temperature Management, we continue to ask for case reports on cases that are extremely unexpected, so people can read about them and that might be a possibility for their future patient as well.

\section{Key References from Panel Participants}

Chavez JF, Doll JA, Mediratta A, Maffessanti F, Friant J, Paul JD, Blair JE, Nathan S, Jolly N, Shah AP. Factors associated with the use of drug-eluting stents in patients presenting with acute ST-segment elevation myocardial infarction. Cardiol Res Pract 2015;2015:528753.

Czobor P, Venturini JM, Parikh KS, Retzer EM, Friant J, Jeevanandam V, Russo MJ, Uriel N, Paul JD, Blair JE, Nathan S, Shah AP. Sequential organ failure assessment score at pre- sentation predicts survival in patients treated with percutaneous veno-arterial extracorporeal membrane oxygenation. J Invasive Cardiol 2016;28:133-138.

Dragancea I, Wise MP, Al-Subaie N, Cranshaw J, Friberg H, Glover G, Pellis T, Rylance R, Walden A, Nielsen N, Cronberg T; TTM trial investigators. Protocol-driven neurological prognostication and withdrawal of life-sustaining therapy after cardiac arrest and targeted temperature management. Resuscitation 2017;117:50-57.

Fisk CA, Olsufka M, Yin L, McCoy AM, Latimer AJ, Maynard C, Nichol G, Larsen J, Cobb LA, Sayre MR. Lower-dose epinephrine administration and out-of-hospital cardiac arrest outcomes. Resuscitation 2018;124:43-48.

Glover GW, Thomas RM, Vamvakas G, Al-Subaie N, Cranshaw J, Walden A, Wise MP, Ostermann M, Thomas-Jones E, Cronberg T, Erlinge D, Gasche Y, Hassager C, Horn J, Kjaergaard J, Kuiper M, Pellis T, Stammet P, Wanscher M, Wetterslev J, Friberg H, Nielsen N. Intravascular versus surface cooling for targeted temperature management after out-of-hospital cardiac arrest-an analysis of the TTM trial data. Crit Care 2016;20:381.

Goslar T, Knafelj R, Radsel P, Fister M, Golicnik A, Steblovnik K, Gorjup V, Noc M. Emergency percutaneous implantation of veno-arterial extracorporeal membrane oxygenation in catheterization laboratory. Eurointervention 2016;12:1465-1472.

Kim F, Maynard C, Nichol G. Prehospital therapeutic hypothermia in patients with out-of-hospital cardiac arrest-reply. JAMA 2014;311:2233-2234.

Lybeck A, Cronberg T, Aneman A, Hassager C, Horn J, Hovdenes J, Kjærgaard J, Kuiper M, Wanscher M, Stammet P, Wise MP, Nielsen N, Ullén S, Friberg H; TTM-trial investigators. Time to awakening after cardiac arrest and the association with target temperature management. Resuscitation 2018;126:166-171.

Maynard C, Longstreth WT Jr., Nichol G, Hallstrom A, Kudenchuk PJ, Rea T, Copass MK, Carlbom D, Deem S, Olsufka M, Cobb LA, Kim F. Effect of prehospital induction of mild hypothermia on 3-month neurological status and 1-year survival among adults with cardiac arrest: long-term followup of a randomized, clinical trial. J Am Heart Assoc 2015;4: $\mathrm{e} 001693$.

Nichol G, Geocadin R, Kurz M, Mooney M. Perspectives on temperature management. Ther Hypothermia Temp Manag 2017;7:8-11.

Nichol G, Strickland W, Shavelle D, Maehara A, Ben-Yehuda O, Genereux P, Dressler O, Parvataneni R, Nichols M, McPherson J, Barbeau G, Laddu A, Elrod JA, Tully GW, Ivanhoe R, Stone GW; VELOCITY Investigators. Prospective, multicenter, randomized, controlled pilot trial of peritoneal hypothermia in patients with ST-segment-elevation myocardial infarction. Circ Cardiovasc Interv 2015;8:e001965.

Nielsen N, Wetterslev J, Cronberg T, Erlinge D, Gasche Y, Hassager C, Horn J, Hovdenes J, Kjaergaard J, Kuiper M, Pellis T, Stammet P, Wanscher M, Wise MP, Åneman A, Al-Subaie N, Boesgaard S, Bro-Jeppesen J, Brunetti I, Bugge JF, Hingston CD, Juffermans NP, Koopmans M, Køber L, Langørgen J, Lilja G, Møller JE, Rundgren M, Rylander C, Smid O, Werer C, Winkel P, Friberg H; TTM Trial Investigators. Targeted temperature management at $33^{\circ} \mathrm{C}$ versus $36^{\circ} \mathrm{C}$ after cardiac arrest. N Engl J Med 2013; 369:2197-2206.

Oddo M, Friberg H. Neuroprognostication after cardiac arrest in the light of targeted temperature management. Curr Opin Crit Care 2017;23:244-250. 
Otani T, Sawano H, Natsukawa T, Matsuoka R, Nakashima T, Takahagi M, Hayashi Y. D-dimer predicts bleeding complication in out-of-hospital cardiac arrest resuscitated with extracorporeal membrane oxygenation. Am J Emerg Med 2017; [Epub ahead of print]; DOI: 10.1016/j.ajem.2017.11.016.

Pollack RA, Brown SP, Rea T, Aufderheide T, Barbic D, Buick JE, Christenson J, Idris AH, Jasti J, Kampp M, Kudenchuk P, May S, Muhr M, Nichol G, Ornato JP, Sopko G, Vaillancourt C, Morrison L, Weisfeldt M; ROC Investigators. Impact of bystander automated external defibrillator use on survival and functional outcomes in shockable observed public cardiac arrests. Circulation 2018;137:2104-2113.

Stammet P, Dankiewicz J, Nielsen N, Fays F, Collignon O, Hassager C, Wanscher M, Undèn J, Wetterslev J, Pellis T, Aneman A, Hovdenes J, Wise MP, Gilson G, Erlinge D, Horn J, Cronberg T, Kuiper M, Kjaergaard J, Gasche Y, Devaux Y, Friberg H; Target Temperature Management after Out-ofHospital Cardiac Arrest (TTM) trial investigators. Protein S100 as outcome predictor after out-of-hospital cardiac arrest and targeted temperature management at $33^{\circ} \mathrm{C}$ and $36^{\circ} \mathrm{C}$. Crit Care 2017;21:153.
Starks MA, Schmicker RH, Peterson ED, May S, Buick JE, Kudenchuk PJ, Drennan IR, Herren H, Jasti J, Sayre M, Stub D, Vilke GM, Stephens SW, Chang AM, Nuttall J, Nichol G; Resuscitation Outcomes Consortium. (ROC) Association of neighborhood demographics with out-of-hospital cardiac arrest treatment and outcomes: where you live may matter. JAMA Cardiol 2017;2:1110-1118.

Steblovnik K, Blinc A, Bozic Mijovski M, Fister M, Mikuz U, Noc M. Ticagrelor versus clopidogrel in comatose survivors of out-of-hospital cardiac arrest undergoing percutaneous coronary intervention and hypothermia: a randomized study. Circulation 2016;134:2128-2130.

Stöckl M, Testori C, Sterz F, Holzer M, Weiser C, Schober A, Nichol G, Frossard M, Herkner H, Kechvar J, Losert H. Continuous versus intermittent neuromuscular blockade in patients during targeted temperature management after resuscitation from cardiac arrest-A randomized, double blinded, double dummy, clinical trial. Resuscitation 2017;120:14-19.

Valenzuela T, Harrell AJ, Nichol G. Improving outcomes after out-of-hospital cardiac arrest. JAMA Cardiol 2017;2:11831184. 